\title{
A Nonlinear Elliptic PDE with Two Sobolev-Hardy Critical Exponents
}

\author{
YanYan Li*and Chang-Shou Lin
}

\begin{abstract}
In this paper, we consider the following PDE involving two Sobolev-Hardy critical exponents,

$$
\left\{\begin{array}{l}
\Delta u+\lambda \frac{u^{2^{*}\left(s_{1}\right)-1}}{|x|^{s_{1}}}+\frac{u^{2^{*}\left(s_{2}\right)-1}}{|x|^{s_{2}}}=0 \text { in } \Omega, \\
u=0 \quad \text { on } \Omega,
\end{array}\right.
$$

where $0 \leq s_{2}<s_{1} \leq 2,0 \neq \lambda \in \mathbb{R}$ and $0 \in \partial \Omega$. The existence (or nonexistence) for least-energy solutions has been extensively studied when $s_{1}=0$ or $s_{2}=0$. In this paper, we prove that if $0<s_{2}<s_{1}<2$ and the mean curvature of $\partial \Omega$ at $0 H(0)<0$, then (0.1) has a least-energy solution. Therefore, this paper has completed the study of (0.1) for the least-energy solutions. We also prove existence or nonexistence of positive entire solutions of (0.1) with $\Omega=\mathbb{R}_{+}^{N}$ under different situations of $s_{1}, s_{2}$ and $\lambda$.
\end{abstract}

\section{Introduction}

Let $0 \leq s \leq 2,2^{*}(s)=\frac{2(N-s)}{N-2}$ and $L^{2^{*}(s)}\left(\frac{d x}{|x|^{s}}\right)$ denote the space of $f$ with $\int|f|^{2^{*}(s)} \frac{d x}{|x|^{s}}<$ $+\infty$. It is well known that the inclusion $H_{0}^{1}(\Omega) \hookrightarrow L^{2^{*}(s)}\left(\frac{d x}{|x|^{s}}\right)$ is a family of non-compact embeddings. In this paper, we want to study the combined effect of two such Sobolev-Hardy critical exponents on a nonlinear partial differential equation. More precisely, we consider

$$
\left\{\begin{array}{l}
\Delta u+\lambda \frac{u^{2^{*}\left(s_{1}\right)-1}}{|x|^{s_{1}}}+\frac{u^{2^{*}\left(s_{2}\right)-1}}{|x|^{s_{2}}}=0 \quad \text { in } \Omega, \\
u(x)>0 \text { in } \Omega \quad u(x)=0 \text { on } \partial \Omega
\end{array}\right.
$$

where $0 \leq s_{2}<s_{1} \leq 2$ and $\lambda \in \mathbb{R}$. Throughout the paper, $\Omega$ is a bounded smooth domain in $\mathbb{R}^{N}$ with $0 \in \partial \Omega$.

Our motivation for studying equation (1.1) comes from the celebrated Caffarelli-KohnNirenberg inequality [4]: there exists a constant $C$ such that for any $u \in C_{0}^{\infty}\left(\mathbb{R}^{N}\right)$, the inequality

$$
\int_{\mathbb{R}^{N}}|x|^{-b q} u^{q} d x \leq C \int_{\mathbb{R}^{N}}|x|^{-2 a}|\nabla u|^{2} d x
$$

*Partially supported by NSF grant DMS-0701545. 
holds, where $-\infty<a<\frac{N-2}{2}, 0 \leq b-a \leq 1$ and $q=\frac{2 N}{N-2+2(b-a)}$. Let $D_{a}^{1,2}(\Omega)$ be the completion of $C_{0}^{\infty}(\Omega)$ with the norm $\|u\|_{a}^{2}=\int_{\mathbb{R}^{N}}|x|^{-2 a}|\nabla u|^{2} d x$, and set

$$
S(a, b ; \Omega)=\inf _{u \in D_{a}^{1,2}(\Omega) \backslash\{0\}} \frac{\int_{\Omega}|x|^{-2 a}|\nabla u|^{2} d x}{\left(\int_{\Omega}|x|^{-b q}|u|^{q} d x\right)^{\frac{2}{q}}} .
$$

Naturally, we ask whether the best constant $S(a, b ; \Omega)$ can be attained by some $u \in D_{a}^{1,2}(\Omega) \backslash$ $\{0\}$. For the past twenty years, this problem has been extensively studied. For recent development, we refer the readers to [1, 5, 6, 11, 12, 13, 14, 15, 22, 23, 24, 19] and the references therein.

When $0 \in \partial \Omega$, this problem was first studied by Ghoussoub-Kang [14] and GhoussoubRobert [15], also see [11]. In [11, among other things, Chern and the second author of this paper proved the following theorem.

Theorem A. Suppose $0 \in \partial \Omega$ and the mean curvature $H(0)<0$. Then the best constant $S(a, b ; \Omega)$ can be achieved in $D_{a}^{1,2}(\Omega)$ if $a, b, q$ satisfy one of the following conditions:

(i) $a<b<a+1$ and $N \geq 3$,

(ii) $b=a>0$ and $N \geq 4$.

When $a=0$ and $0<b<1$, Theorem $\mathrm{A}$ was first proved by Ghoussoub and Robert [15]. The proof of Theorem $\mathrm{A}$ in [11] is to make use of a transformation: $u(x)=|x|^{-a} v(x)$. Straightforward computations give

$$
\int_{\Omega}|x|^{-2 a}|\nabla v|^{2} d x=\int_{\Omega}|\nabla u|^{2} d x-\lambda \int_{\Omega} \frac{u^{2}}{|x|^{2}} d x
$$

where $\lambda=a(N-2-a)$. Then $S(a, b ; \Omega)$ is equal to the following best constant:

$$
S_{\lambda}(\Omega)=\inf _{u \in H_{0}^{1}(\Omega) \backslash\{0\}} \frac{\int_{\Omega}|\nabla u|^{2} d x-\lambda \int_{\Omega} \frac{|u|^{2}}{|x|^{2}} d x}{\left(\int_{\Omega} \frac{|u|^{2 *(s)}}{|x|^{s}} d x\right)^{\frac{2}{2^{2}(s)}}},
$$

where $\lambda=a(N-2-a)$ and $s=(b-a) q \in[0,2)$ if $b<a+1$. Note that if $b=a+1$, thus $s=2$ and the question for the best constant is a linear problem. Hence, we always exclude the case $b=a+1$. By (1.2), Theorem $A$ is equivalent to saying that equation (1.1) has a solution provided that either (i) $N \geq 3, \lambda<\left(\frac{N-2}{2}\right)^{2}, 0<s_{2}<s_{1}=2$, or (ii) $N \geq 4$, $0<\lambda<\left(\frac{N-2}{2}\right)^{2}, s_{1}=2$ and $s_{2}=0$.

To study equation (1.1), we consider the nonlinear functional $\Phi$ :

$$
\Phi(u)=\frac{1}{2} \int_{\Omega}|\nabla u|^{2} d x-\frac{\lambda}{p_{1}+1} \int_{\Omega} \frac{\left(u^{+}\right)^{p_{1}+1}}{|x|^{s_{1}}} d x-\frac{1}{p_{1}+1} \int_{\Omega} \frac{\left(u^{+}\right)^{p_{2}+1}}{|x|^{s_{2}}} d x
$$

for $u \in H_{0}^{1}(\Omega)$, where for the simplicity of notations, we let $p_{1}=2^{*}\left(s_{1}\right)-1$ and $p_{2}=2^{*}\left(s_{2}\right)-1$. It is easy to see that there is positive constants $\rho_{0}, c_{0}>0$ such that

$$
\Phi(u) \geq c_{0} \text { if }\|u\|_{H_{0}^{1}}=\rho_{0} .
$$

Note that $p_{2}>p_{1}$ because $s_{1}>s_{2}$. Thus, no matter what the sign of $\lambda$ is, there is $u_{0} \in H_{0}^{1}(\Omega)$ such that $\Phi\left(u_{0}\right) \leq 0$. Set

$$
c_{*}=\inf _{P \in \mathcal{P}} \max _{w \in P} \Phi(w),
$$


where $\mathcal{P}$ is the class of continuous paths in $H_{0}^{1}(\Omega)$ connecting 0 and $u_{0}$. We note that since $p_{2}>p_{1}$, the function $t \rightarrow \Phi(t u)$ has the unique maximum for $t \geq 0$. Furthermore, we have

$$
c_{*}=\inf _{u \in H_{0}^{1}(\Omega)} \max _{u \geq 0, u \neq 0} \Phi(t u) .
$$

It is well-known that due to the non-compact embedding of $H_{0}^{1} \hookrightarrow L^{2^{*}(s)}\left(\frac{d x}{|x|^{s}}\right), \Phi$ does not satisfy the Palais-Smale condition. Therefore, in general $c_{*}$ might not be a critical value for $\Phi$. As usual, if $c_{*}$ is a critical value, and $u$ is a critical point of $\Phi$ with $\Phi(u)=c_{*}$, then $u$ is called a least-energy solution.

When $s_{2}=0$, equation (1.1) becomes

$$
\Delta u+\lambda \frac{u^{2^{*}\left(s_{1}\right)-1}}{|x|^{s_{1}}}+u^{\frac{N+2}{N-2}}=0 \text { in } \Omega .
$$

When $\lambda<0$, the best constant $S_{\lambda}(\Omega)$ of (1.2) always satisfies

$$
S_{\lambda}(\Omega)=S_{0}(\Omega)=S_{N},
$$

where $S_{N}$ is the Sobolev best constant. Thus, $S_{\lambda}(\Omega)$ can not be attained in $H_{0}^{1}(\Omega)$, and as a consequence, $c_{*}$ could not be a critical value of $\Phi$. In fact, for $0 \leq s_{1}<2$, it is not difficult to see that the constant $c_{*}$ of (1.3) is always equal to $\frac{1}{N} S_{N}^{\frac{N}{2}}$ and $c_{*}$ is never a critical value for $\Phi$. Thus, there exist no least-energy solutions for equation (1.4) when $\lambda<0$. However, when $\lambda>0,0<s_{1}<2$ and $s_{2}=0$, the following theorem was proved in [17.

Theorem B. Suppose $N \geq 4$ and $0 \in \partial \Omega$ with $H(0)<0$. Then equation (1.4) has a solution, provided that $\lambda>0$, and $0<s_{1} \leq 2$.

In summary, equation (1.1) has been studied for either $s_{1}=2$ or $s_{2}=0$. The purpose of this paper is to study the remaining cases for equation (1.1). The following is one of our main theorem.

Theorem 1.1. Suppose $\Omega$ is a bounded smooth domain in $\mathbb{R}^{N}, 0 \in \partial \Omega$ and the mean curvature $H(0)<0$. Then equation (1.1) has a least-energy solution if

$$
N \geq 3, \lambda \in \mathbb{R} \text { and } 0<s_{2}<s_{1}<2 .
$$

In principle, the solvability of least energy solutions is closely related to the existence of the entire solutions of equation (1.1), i.e., $\Omega=\mathbb{R}_{+}^{N}$, the upper half-space. The existence of entire solutions on the upper half space has been proved by Bartsch, Peng and Zhang [1] when $0<s_{2}<s_{1}=2$ and $\lambda<\left(\frac{N-2}{2}\right)^{2}$, by Musina 25] when $N \geq 4, s_{2}=0, s_{1}=2$ and $0<\lambda<\left(\frac{N-2}{2}\right)^{2}$, and by Hsia, Lin and Wadade [17] when $s_{2}=0,0<s_{1}<2$ and $\lambda>0$. Close to Theorem 1.1 the following existence of positive entire solutions will be proved in this paper.

Theorem 1.2. Let $N \geq 3,0<s_{2}<s_{1}<2, \lambda \in \mathbb{R}$ and $\Omega=\mathbb{R}_{+}^{N}$. Then equation (1.1) has a least-energy solution $u \in H_{0}^{1}\left(\mathbb{R}_{+}^{N}\right)$.

To complement Theorem[1.2, we prove the following non-existence of entire solutions of (1.1) when $s_{2}=0$ and $\lambda \leq 0$.

Theorem 1.3. Let $\Omega=\mathbb{R}_{+}^{N}, 0<s_{1} \leq 2$ and $\lambda \leq 0$. Suppose $u(x) \in H_{\text {loc }}^{1}\left(\overline{\mathbb{R}_{+}^{N}}\right)$ and $u(x) \geq 0$ is a solution of (1.4). Then $u(x) \equiv 0$. 
We note that if solutions are assumed to be in $H_{0}^{1}\left(\mathbb{R}_{+}^{N}\right)$, Theorem 1.3 with $s_{1}=2$ has been proved in [17. The authors of [17] employed the method of moving planes to prove Theorem 1.3. where the behavior of $u$ at $\infty$ is needed. One way to find asymptotic behavior is to apply the Kelvein transform to $u$ :

$$
\hat{u}(y)=\left(\frac{1}{|y|}\right)^{N-2} u\left(\frac{y}{|y|^{2}}\right) \quad \text { for } \quad|y|<1 .
$$

It is a straightforward computation to show that $\hat{u}(y)$ satisfies

$$
\Delta \hat{u}+\lambda \frac{\hat{u}^{2^{*}\left(s_{1}\right)-1}(y)}{|y|^{s_{1}}}+\hat{u}^{\frac{N+2}{N-2}}(y)=0 \quad \text { in } \quad B_{1} \cap \mathbb{R}_{+}^{N} .
$$

But $\hat{u}$ is no longer contained in $H_{l o c}^{1}\left(\overline{\mathbb{R}_{+}^{N}}\right)$, i.e., the integration of $\nabla \hat{u}$ might be $+\infty$ in any neighborhood of 0 . In this case, the origin 0 is called a nonremovable singularity of $\hat{u}$. It is a really interesting question: What is the asymptotic behavior of $\hat{u}$ near the singularity? Previously, this kind of problems have been studied:

$$
\Delta u+g(y, u)+u^{\frac{N+2}{N-2}}=0 \quad \text { in } 0<|y|<1 .
$$

Under the monotonicity assumption of $u$ :

$$
g(y, t) t^{-\frac{N+2}{N-2}} \text { is decreasing for large } t>0,
$$

it was proved that $u(y)=O\left(|y|^{-\frac{N-2}{2}}\right)$ near 0 . See [7, 8, 9, 10, For our case,

$$
g(y, u)=\lambda \frac{u^{2^{*}\left(s_{1}\right)-1}}{|y|^{s_{1}}} \quad \text { and } \quad \lambda<0 .
$$

Then $g(y, t) t^{-\frac{N+2}{N-2}}$ is increasing in $t>0$. Hence, the methods in [7, 8, 9, 10, can not work for our nonlinearity. We should address this asymptotic problem later.

Our proof of Theorem 1.3 employs the idea of the method of moving spheres, a variant of the method of moving planes. The method of moving planes has been developed through the works by A.D. Alexandrov, Serrin [26, and Gidas, Ni and Nirenberg [16]. Here, we will not require any assumption on the behavior of solutions at $\infty$, by taking some advantage of the upper half space $\mathbb{R}_{+}^{N}$, while compared to $\mathbb{R}^{N}$. We think this proof might be useful in other problems also. See [21, 20, 18] for some related results.

This paper is organized as follows. In Section 2, we will prove Theorem 1.3 and a generalization of it. In Section 3, we will employ a blowing-up argument to prove Theorem 1.2 This kind of arguments have been developed for studying the nonlinear equation involving the Sobolev critical exponent, see 17, 8, 9, 10, 19. The existence of least-energy solutions of equation (1.1) with $0<s_{2}<s_{1}<2$ are obtained in Section 4. In final section, we discuss a perturbed equation of equation (1.1) for the case $\lambda<0,0=s_{2}<s_{1}<2$.

\section{Nonexistence of Entire Solutions}

In this section, we begin with a proof of Theorem 1.3 We first make a remark about regularity of $u(x)$. It is shown that $u \in C^{\alpha}\left(\overline{\mathbb{R}_{+}^{N}}\right)$, for any $\alpha \in(0,1)$. For a proof, see [11 and 17.

If $u=0$ at some point of $\mathbb{R}_{+}^{N}$, then $u \equiv 0$ by the strong maximum principle. Hence, we will always assume that

$$
u(x)>0 .
$$

We will prove a lemma below. 
Lemma 2.1. Let $u(x)$ be a positive solution of equation (1.4). Suppose $u \in H_{l o c}^{1}\left(\overline{\mathbb{R}_{+}^{N}}\right)$. Then $\frac{\partial u}{\partial x_{N}}>0$ in $\mathbb{R}_{+}^{N}$.

Before giving a proof of Lemma2.1 we apply Lemma 2.1 to prove Theorem 1.3 ,

Proof of Theorem 1.3. Suppose $u(x)$ is a positive solution of equation (1.4).

We claim $u$ is uniformly bounded in any compact set of $\mathbb{R}^{N}$. Suppose the contrary, then there exist $\bar{x}_{i} \in \mathbb{R}^{N}$, such that

$$
u\left(\bar{x}_{i}\right) \rightarrow \infty \quad \text { as } i \rightarrow \infty .
$$

By the monotonicity of $u$ in $x_{N}$-direction, we may assume that

$$
\left|\bar{x}_{i}\right| \rightarrow \infty
$$

Consider

$$
v_{i}(x)=\left(1-\left|x-\bar{x}_{i}\right|\right)^{\frac{N-2}{2}} u(x), \quad\left|x-\bar{x}_{i}\right| \leq 1 .
$$

For some $\left|x_{i}-\bar{x}_{i}\right|<1$,

$$
v_{i}\left(x_{i}\right)=\max _{\left|x-\bar{x}_{i}\right| \leq 1} v_{i}(x),
$$

here we have used the fact that $v_{i}(x)=0$ for $\left|x-\bar{x}_{i}\right|=1$.

Let

$$
\sigma_{i}=\frac{1}{2}\left(1-\left|x_{i}-\bar{x}_{i}\right|\right)>0 .
$$

Then

$$
\left(2 \sigma_{i}\right)^{\frac{N-2}{2}} u\left(x_{i}\right)=v_{i}\left(x_{i}\right) \geq v_{i}\left(\bar{x}_{i}\right)=u\left(\bar{x}_{i}\right) \rightarrow \infty .
$$

It follows that

$$
R_{i}:=\sigma_{i} u\left(x_{i}\right)^{\frac{2}{N-2}} \rightarrow \infty .
$$

Since

$$
v_{i}\left(x_{i}\right) \geq v_{i}(x) \geq \sigma_{i}^{\frac{N-2}{2}} u(x), \quad \forall x \in B_{\sigma_{i}}\left(x_{i}\right),
$$

we see that

$$
u(x) \leq 2^{\frac{N-2}{2}} u\left(x_{i}\right), \quad \forall x \in B_{\sigma_{i}}\left(x_{i}\right) .
$$

Consider

$$
w_{i}(y):=\frac{1}{u\left(x_{i}\right)} u\left(x_{i}+\frac{y}{u\left(x_{i}\right)^{\frac{2}{N-2}}}\right), \quad|y|<R_{i}=\sigma_{i} u_{i}\left(x_{i}\right)^{\frac{2}{N-2}} \rightarrow \infty .
$$

Then

$$
w_{i}(0)=1, \quad \text { and } \quad w_{i}(y) \leq 2^{\frac{N-2}{2}}, \quad \forall|y|<R_{i} .
$$

Using the equation satisfied by $u_{i}$, we have

$$
\Delta w_{i}(y)-\frac{1}{u\left(x_{i}\right)^{\frac{2 s_{1}}{N-2}}} \frac{w_{i}(y)^{2^{*}\left(s_{1}\right)-1}}{\left|x_{i}+\frac{y}{u\left(x_{i}\right)^{\frac{2}{N-2}}}\right|^{s_{1}}}+w_{i}(y)^{\frac{N+2}{N-2}}=0, \quad|y|<R_{i} .
$$

Since $\left|\bar{x}_{i}\right| \rightarrow \infty$, it is clear that

$$
\left|x_{i}+\frac{y}{u\left(x_{i}\right)^{\frac{2}{N-2}}}\right| \geq\left|\bar{x}_{i}\right|-\left|x_{i}-\bar{x}_{i}\right|-\sigma_{i} \geq\left|\bar{x}_{i}\right|-2 \rightarrow \infty, \quad \text { uniformly for }|y|<R_{i} .
$$


Given the bound of $w_{i}$, we know from standard elliptic estimates that on every compact subset of $\mathbb{R}^{N},\left\{w_{i}\right\}$ is bounded in $C^{3}$ norm. After passing to a subsequence, we have,

$$
w_{i} \rightarrow w \quad \text { in } C_{l o c}^{2}\left(\overline{\mathbb{R}_{+}^{N}}\right) .
$$

Given the above estimates, and the equation of $w_{i}$, we have

$$
\Delta w+w^{\frac{N+2}{N-2}}=0, \quad \text { on } \mathbb{R}^{N},
$$

and

$$
w(0)=1, \quad w \geq 0 \quad \text { on } \mathbb{R}^{N} .
$$

By the strong maximum principle, $w>0$ on $R^{N}$.

By the classification theorem of Caffarelli-Gidas-Spruck,

$$
w(y)=C_{N}\left(\frac{\mu}{1+\mu^{2}\left|y-y_{0}\right|^{2}}\right)^{\frac{N-2}{2}},
$$

where $\mu>0$ and $y_{0} \in \mathbb{R}^{N}$.

But we know from the monotonicity of $w_{i}, w$ must be monotone in $y_{N}$-direction. This is a contradiction and the claim is proved.

Let $u_{j}\left(x^{\prime}, x_{N}\right)=u\left(x^{\prime}, x_{N}+r_{j}\right)$ where $r_{j}<r_{j+1} \rightarrow+\infty$ as $j \rightarrow+\infty$. By Lemma2.1 $u_{j}(x)<u_{j+1}(x)$. Since $u(x)$ is uniformly bounded, $u_{j}(x) \rightarrow u_{\infty}(x)$ in $C_{l o c}^{2}\left(\mathbb{R}^{N}\right)$, where $u_{\infty}(x)$ is a positive solution to

$$
\Delta u_{\infty}+u_{\infty}^{\frac{N+2}{N-2}}=0 \text { in } \mathbb{R}^{N} .
$$

Again, Lemma2.1 yields a contradiction to (2.2). Hence, the proof of Theorem 1.3 is complete.

Proof of Lemma 2.1. The proof uses the method of moving spheres, a variant of the method of moving planes which are developed through the works of Alexandrov, Serrin [26, and Gidas, Ni and Nirenberg [16. We also make use of the "narrow domain idea" from Berestycki and Nirenberg [2].

Define

$$
x_{R}:=(0, \cdots, 0,-R) .
$$

Let

$$
u_{x_{R}, \lambda}(y):=\left(\frac{\lambda}{\left|y-x_{R}\right|}\right)^{N-2} u\left(x_{R}+\frac{\lambda^{2}\left(y-x_{R}\right)}{\left|y-x_{R}\right|^{2}}\right)
$$

be the Kelvin transformation of $u$ with respect to the ball $B_{\lambda}\left(x_{R}\right)$ with center $x_{R}$ and radius $\lambda>0$. By direct computations, we have for $y \in B_{\lambda}\left(x_{R}\right) \cap \mathbb{R}_{+}^{N}$,

$$
\Delta u_{x_{R}, \lambda}(y)-\left(\frac{\lambda}{\left|y-x_{R}\right|}\right)^{2 s} \frac{u_{x_{R}, \lambda}^{2^{*}(s)-1}(y)}{\left|x_{R}+\frac{\lambda^{2}\left(y-x_{R}\right)}{y-x_{R}}\right|^{s}}+u_{x_{R}, \lambda}^{\frac{N+2}{N-2}}=0 .
$$

We want to show that

$$
u_{x_{R}, \lambda}(y) \geq u(y) \quad \forall y \in B_{\lambda}\left(x_{R}\right) \cap \mathbb{R}_{+}^{N}, \forall \lambda>R .
$$

To prove (2.3), we first claim

$$
\left(\frac{\lambda}{\left|y-x_{R}\right|}\right)^{2 s} \frac{1}{\left|x_{R}+\frac{\lambda^{2}\left(y-x_{R}\right)}{\left|y-x_{R}\right|^{2}}\right|^{s}} \leq \frac{1}{|y|^{s}},
$$


for $y \in B_{\lambda}\left(x_{R}\right) \cap \mathbb{R}_{+}^{N}, \forall \lambda>R$.

For $y \in B_{\lambda}\left(x_{R}\right) \cap \mathbb{R}_{+}^{N}$, we write

$$
\frac{y-x_{R}}{\left|y-x_{R}\right|}=\theta=\left(\theta_{1}, \cdots, \theta_{N}\right), \quad\left|y-x_{R}\right|=r .
$$

Then

$$
\mu_{1}(\theta)<r<\lambda,
$$

where $\mu_{1}(\theta)$ is determined by

$$
x_{R}+\mu_{1}(\theta) \theta \in \partial \partial \mathbb{R}_{+}^{N} .
$$

Namely,

$$
\mu_{1}(\theta)=\frac{R}{\theta_{N}} .
$$

(2.4) is equivalent to

$$
\left(\frac{\lambda}{r}\right)^{2 s} \frac{1}{\left|x_{R}+\frac{\lambda^{2}}{r} \theta\right|^{s}} \leq \frac{1}{\left|x_{R}+r \theta\right|^{s}} .
$$

This is equivalent to

$$
\left(\frac{\lambda^{2}}{r}\right)^{2} \frac{1}{\left|x_{R}+\frac{\lambda^{2}}{r} \theta\right|^{2}} \leq r^{2} \frac{1}{\left|x_{R}+r \theta\right|^{2}} .
$$

For $r$ satisfying (2.5), we have

$$
\frac{\lambda^{2}}{R}>\frac{\lambda^{2}}{r}>r>\mu_{1}(\theta) .
$$

Let

$$
\eta(\mu):=\mu^{2} \frac{1}{\left|x_{R}+\mu \theta\right|^{2}}, \quad \mu>\mu_{1}(\theta) .
$$

In order to prove (2.6), we only need to prove

$$
\eta^{\prime}(\mu) \leq 0, \quad \mu_{1}(\theta)<\mu<\frac{\lambda^{2}}{R} .
$$

This follows from the following calculations, for $\mu>\mu_{1}(\theta)$,

$$
\begin{aligned}
\left|x_{R}+\mu \theta\right|^{4} \eta^{\prime}(\mu) & =2 \mu\left|x_{R}+\mu \theta\right|^{2}-\mu^{2} \frac{d}{d \mu}\left(\left|x_{R}+\mu \theta\right|^{2}\right) \\
& =2 \mu R \theta_{N}\left(\mu_{1}-\mu\right)<0 .
\end{aligned}
$$

We have proved (2.7), and therefore proved (2.4). It follows that

$$
-\Delta u_{x_{R}, \lambda}+\frac{1}{|y|^{s}} u_{x_{R}, \lambda}^{2(s)-1} \geq u_{x_{R}, \lambda}(y)^{\frac{N+2}{N-2}}, \quad \text { in } B_{\lambda}\left(x_{R}\right) \cap R_{+}^{n} .
$$

Thus

$$
-\Delta\left(u_{x_{R}, \lambda}-u\right)+\frac{1}{|y|^{s}}\left(u_{x_{R}, \lambda}^{2^{*}(s)-1}-u^{2^{*}(s)-1}\right) \geq u_{x_{R}, \lambda}^{\frac{N+2}{N-2}}-u^{\frac{N+2}{N-2}}, \quad \text { in } B_{\lambda}\left(x_{R}\right) \cap \mathbb{R}_{+}^{N} .
$$

Write

$$
w_{\lambda}=u_{x_{R}, \lambda}-u, \quad w_{\lambda}^{-}=\max \left\{0,-w_{\lambda}\right\} .
$$


We first require that $R<\lambda_{0}(R)<2 R$, then for $R<\lambda<\lambda_{0}(R)$, we have

$$
\left|x_{R}+\frac{\lambda^{2}\left(y-x_{R}\right)}{\left|y-x_{R}\right|^{2}}\right| \leq\left|x_{R}\right|+\frac{\lambda^{2}}{R} \leq 5 R, \quad \forall y \in B_{\lambda}\left(x_{R}\right) \cap \mathbb{R}_{+}^{N} .
$$

Multiply $w_{\lambda}^{-}$to the inequality (2.8) and integrate by parts on $B_{\lambda}\left(x_{R}\right) \cap \mathbb{R}_{+}^{N}$, we have, using $w_{\lambda} \geq 0$ on $\partial\left(B_{\lambda}\left(x_{R}\right) \cap \mathbb{R}_{+}^{N}\right)$,

$$
\begin{aligned}
& \int_{B_{\lambda}\left(x_{R}\right) \cap \mathbb{R}_{+}^{N}}\left|\nabla w_{\lambda}^{-}\right|^{2} d y \\
\leq & \int_{B_{\lambda}\left(x_{R}\right) \cap \mathbb{R}_{+}^{N}}\left(\left|\nabla w_{\lambda}^{-}\right|^{2}-\frac{1}{|y|^{s}}\left(u_{x_{R}, \lambda}^{2^{*}(s)-1}-u^{2^{*}(s)-1}\right) w_{\lambda}^{-} d y\right. \\
\leq & \int_{B_{\lambda}\left(x_{R}\right) \cap \mathbb{R}_{+}^{N}}\left(u_{x_{R}, \lambda}^{\frac{N+2}{N-2}}-u^{\frac{N+2}{N-2}}\right) w_{\lambda}^{-} d y \\
\leq & \frac{N+2}{N-2} \int_{B_{\lambda}\left(x_{R}\right) \cap \mathbb{R}_{+}^{N}}\left(\max \left\{u_{x_{R}, \lambda}, u\right\}\right)^{\frac{4}{N-2}}\left(w_{\lambda}^{-}\right)^{2} d y \\
\leq & \frac{N+2}{N-2} \sup _{B_{5 R}(0) \cap \mathbb{R}_{+}^{N}} u \int_{B_{\lambda}\left(x_{R}\right) \cap \mathbb{R}_{+}^{N}}\left(w_{\lambda}^{-}\right)^{2} d y \\
\leq & C(N)\left|B_{\lambda}\left(x_{R}\right) \cap \mathbb{R}_{+}^{N}\right|^{\frac{2}{N}}\left\|w_{\lambda}^{-}\right\|_{L^{\frac{2 N}{N-2}}}^{2}\left(B_{\lambda}\left(x_{R}\right) \cap \mathbb{R}_{+}^{N}\right) \\
\leq & C(N)\left|B_{\lambda}\left(x_{R}\right) \cap \mathbb{R}_{+}^{N}\right|^{\frac{2}{N}} \int_{B_{\lambda}\left(x_{R}\right) \cap \mathbb{R}_{+}^{N}}\left|\nabla w_{\lambda}^{-}\right|^{2} d y .
\end{aligned}
$$

Now we can choose $\lambda_{0}(R)>R$ but very close to $R$, then $\left|B_{\lambda}\left(x_{R}\right) \cap \mathbb{R}_{+}^{N}\right|$ is small, and we have

$$
\int_{B_{\lambda}\left(x_{R}\right) \cap \mathbb{R}_{+}^{N}}\left|\nabla w_{\lambda}^{-}\right|^{2} d y \leq \frac{1}{2} \int_{B_{\lambda}\left(x_{R}\right) \cap \mathbb{R}_{+}^{N}}\left|\nabla w_{\lambda}^{-}\right|^{2} d y .
$$

This implies $\nabla w_{\lambda}^{-}=0$ in $B_{\lambda}\left(x_{R}\right) \cap \mathbb{R}_{+}^{N}$ and therefore, since $w_{\lambda}^{-}=0$ on $\partial B_{\lambda}\left(x_{R}\right) \cap \mathbb{R}_{+}^{N}$, $w_{\lambda}^{-}=0$ in $B_{\lambda}\left(x_{R}\right) \cap \mathbb{R}_{+}^{N}$. Step 1 is established.

Define

$$
\bar{\lambda}(R):=\sup \left\{\mu \mid \mu>R, \text { and } u_{x_{R}, \lambda}(y) \geq u(y), \forall y \in B_{\lambda}\left(x_{R}\right) \cap \mathbb{R}_{+}^{N}, \forall R<\lambda<\mu\right\} .
$$

By Step $1, \bar{\lambda}(R)$ is well defined and $R<\bar{\lambda}(R) \leq \infty$.

Step 2. $\bar{\lambda}(R)=\infty$ for all $R>0$.

We establish Step 2 by contradiction. Suppose that $\bar{\lambda} \equiv \bar{\lambda}(R)<\infty$ for some $R>0$. Then

$$
u_{x_{R}, \bar{\lambda}}(y) \geq u(y), \forall y \in B_{\bar{\lambda}}\left(x_{R}\right) \cap \mathbb{R}_{+}^{N} .
$$

Since $u_{x_{R}, \bar{\lambda}}>u$ on $B_{\bar{\lambda}}\left(x_{R}\right) \cap \partial \mathbb{R}_{+}^{N}$, we have, by the strong maximum principle,

$$
u_{x_{R}, \bar{\lambda}}(y)>u(y), \forall y \in B_{\bar{\lambda}}\left(x_{R}\right) \cap \mathbb{R}_{+}^{N} .
$$

For $\delta>0$ small, and the value to be fixed below, let

$$
K:=\left\{y \in B_{\bar{\lambda}}\left(x_{R}\right) \cap \mathbb{R}_{+}^{N} \mid \operatorname{dist}\left(y, \partial\left(B_{\bar{\lambda}}\left(x_{R}\right) \cap \mathbb{R}_{+}^{N}\right)\right) \geq \delta\right\} .
$$


Then

$$
b:=\min _{K} w_{\bar{\lambda}}>0,
$$

where we have used the notation (2.9).

Consider $\bar{\lambda}<\lambda<\bar{\lambda}+\epsilon$, where the value of $\epsilon=\epsilon(\delta)<\delta$ is chosen so that

$$
w_{\lambda}>\frac{b}{2}, \quad \text { on } K, \quad \forall \bar{\lambda}<\lambda<\bar{\lambda}+\epsilon .
$$

Multiplying (2.8) by $w_{\lambda}^{-}$and integrating by parts on $\left(B_{\lambda}\left(x_{R}\right) \cap \mathbb{R}_{+}^{N}\right) \backslash K$ leads to, as before,

$$
\begin{aligned}
& \int_{\left(B_{\lambda}\left(x_{R}\right) \cap \mathbb{R}_{+}^{N}\right) \backslash K}\left|\nabla w_{\lambda}^{-}\right|^{2} d y \\
& \leq \int_{\left(B_{\lambda}\left(x_{R}\right) \cap \mathbb{R}_{+}^{N}\right) \backslash K}\left(\left|\nabla w_{\lambda}^{-}\right|^{2}-\frac{1}{|y|^{s}}\left(u_{x_{R}, \lambda}^{2^{*}(s)-1}-u^{2^{*}(s)-1}\right) w_{\lambda}^{-} d y\right. \\
& \leq C\left|\left(B_{\lambda}\left(x_{R}\right) \cap \mathbb{R}_{+}^{N}\right) \backslash K\right|^{\frac{2}{N}} \int_{\left(B_{\lambda}\left(x_{R}\right) \cap \mathbb{R}_{+}^{N}\right) \backslash K}\left|\nabla w_{\lambda}^{-}\right|^{2} d y .
\end{aligned}
$$

Now we can fix the value of $\delta$ so that $C\left|\left(B_{\lambda}\left(x_{R}\right) \cap \mathbb{R}_{+}^{N}\right) \backslash K\right|^{\frac{2}{N}}<\frac{1}{2}$, and we obtain as before $w_{\lambda}^{-}=0$ on $\left(B_{\lambda}\left(x_{R}\right) \cap \mathbb{R}_{+}^{N}\right) \backslash K$, i.e.

$$
u_{x_{R}, \lambda}(y) \geq u(y), \forall y \in\left(B_{\lambda}\left(x_{R}\right) \cap \mathbb{R}_{+}^{N}\right) \backslash K, \quad \forall \bar{\lambda}<\lambda<\bar{\lambda}+\epsilon .
$$

This and (2.10) contradicts to the definition of $\bar{\lambda}(R)$. Step 2 is established.

By Step 2, we have

$$
u_{x_{R}, R+a}(y) \geq u(y), \quad \forall y \in B_{R+a}\left(x_{R}\right) \cap \mathbb{R}_{+}^{N}, \forall R, a>0 .
$$

It follows, for every $y \in \mathbb{R}_{+}^{N}$, and every $a>y_{n}$,

$$
u(y) \leq \lim _{R \rightarrow \infty} u_{x_{R}, R+a}(y)=u\left(y_{1}, \cdots, y_{N-1}, 2 a-y_{N}\right) .
$$

The above implies

$$
u\left(y_{1}, \cdots, y_{N-1}, s\right) \leq u\left(y_{1}, \cdots, y_{N-1}, t\right), \quad \forall 0<s<t .
$$

We have proved

$$
\frac{\partial u}{\partial x_{N}} \geq 0, \quad \text { in } \mathbb{R}_{+}^{N}
$$

Applying $\frac{\partial}{\partial x_{N}}$ to the equation of $u$ leads to

$$
-\Delta\left(\frac{\partial u}{\partial x_{N}}\right)+\left(\frac{2^{*}(s)-1}{|x|^{s}} u^{2^{*}(s)-2}-\frac{N+2}{N-2} u^{\frac{4}{n-2}}\right)\left(\frac{\partial u}{\partial x_{N}}\right)+\frac{\partial}{\partial x_{N}}\left(\frac{1}{|x|^{s}}\right) u=0, \quad \text { in } \mathbb{R}_{+}^{N} .
$$

By the strong maximum principle, we have $\frac{\partial u}{\partial x_{n}}$ is always zero or strictly positive. But $u=0$ on the boundary on $\mathbb{R}_{+}^{N}$ and positive in $\mathbb{R}_{+}^{N}$, so we must have $\frac{\partial u}{\partial x_{n}}>0$ in $\mathbb{R}_{+}^{N}$. Lemma 2.1 is established.

The main theorem in this section is the following generalization of Theorem 1.3. 
Theorem 2.2. Let $s_{i} \in(0,2], P_{i} \in \mathbb{R}^{N-1}$ and let $u(x) \geq 0$ be a solution of

$$
\left\{\begin{array}{l}
\Delta u-\sum_{i=1}^{l} \frac{u^{2^{*}\left(s_{i}\right)-1}}{\left|x-P_{i}\right|^{s_{i}}}+u^{\frac{N+2}{N-2}}=0 \quad \text { in } \mathbb{R}_{+}^{N}, \\
u(x)=0 \text { on } \partial \mathbb{R}_{+}^{N} .
\end{array}\right.
$$

Suppose $u \in L^{\infty}\left(\mathbb{R}_{+}^{N}\right) \cap H_{\text {loc }}^{1}\left(\overline{\mathbb{R}_{+}^{N}}\right)$. Then $u(x) \equiv 0$.

Proof. The main step is to show that $\frac{\partial u}{\partial x} \geq 0$ as did in Lemma 2.1. This second proof could work for the general situation of (2.12), but the boundedness of $u$ is required! The proof is divided into several steps.

Step 1. $u(x) \rightarrow 0$ as $|x| \rightarrow+\infty$. Suppose not. We may assume there are $x_{j} \rightarrow+\infty$, $u\left(x_{j}\right) \geq C>0$ for some positive constant $C$. Let $u_{j}(x)=u\left(x+x_{j}\right)$. By elliptic estimates, $u_{j}(x)$ is bounded in $C^{2}$ in any compact set of $\overline{\mathbb{R}_{+}^{N}}$. By passing to a subsequence, we may assume $u_{j}(x) \rightarrow u(x)$ in $C_{l o c}^{2}\left(\overline{\mathbb{R}_{+}^{N}}\right)$ and $u(x)$ satisfies

$$
\left\{\begin{array}{l}
\Delta u(x)+u^{\frac{N+2}{N-2}}=0 \\
u \equiv 0 \quad \text { on } \partial \mathbb{R}_{+}^{N}
\end{array} \quad \text { in } \mathbb{R}_{+}^{N}\right.
$$

But it is well-known that 2.13) has no positive solutions. Thus, $u \equiv 0$ in $\mathbb{R}_{+}^{N}$ which contradicts to $u(0) \geq C>0$. So, Step 1 is proved.

Step 2. We claim for any $\lambda>0$,

$$
u\left(y^{\lambda}\right)>u(y) \quad \text { for } x \in \Sigma_{\lambda}=\left\{\left(y_{1}, y_{2}, \cdots, y_{N}\right) \mid 0 \leq y_{N}<\lambda\right\}
$$

where $y^{\lambda}=\left(y_{1}, \cdots, y_{N-1}, 2 \lambda-y_{N}\right)$. This step is a standard application of the method of moving planes. We give a sketch of proofs for the sake of completeness. Let

$$
w_{\lambda}(y)=u\left(y^{\lambda}\right)-u(y) .
$$

Then we have

$$
\begin{aligned}
\Delta w_{\lambda}(y) & -\sum_{j=1}^{l} \frac{1}{\left|y-P_{j}\right|^{s_{j}}}\left(u^{2^{*}\left(s_{j}\right)-1}\left(y^{\lambda}\right)-u^{2^{*}\left(s_{j}\right)-1}(y)\right)+u^{\frac{N+2}{N-2}}\left(y^{\lambda}\right)-u^{\frac{N+2}{N-2}}(y) \\
& =\sum_{j=1}^{l}\left(\frac{1}{\left|y^{\lambda}-P_{j}\right|^{s_{j}}}-\frac{1}{|y|^{s_{j}}}\right) u^{2^{*}\left(s_{j}\right)-1}\left(y^{\lambda}\right) \leq 0 \quad \text { in } \Sigma_{\lambda} .
\end{aligned}
$$

Thus $w_{\lambda}(y)$ satisfies

$$
\Delta w_{\lambda}(y)+\left(C_{1}(y)+C_{2}(y)\right) w_{\lambda}(y) \leq 0 \quad \text { in } \Sigma_{\lambda},
$$

where

$$
C_{1}(y) \leq 0, \quad \text { and } \quad C_{2}(y)=\frac{u^{\frac{N+2}{N-2}}\left(y^{\lambda}\right)-u^{\frac{N+2}{N-2}}(y)}{u\left(y^{\lambda}\right)-u(y)} .
$$

By Step $1, C_{2}(y)=o(1)$ as $|y| \rightarrow+\infty$ and $y \in \Sigma_{\lambda}$. 
To prove $w_{\lambda}(y)>0$ in $\Sigma_{\lambda}$ for $\lambda$ small, we consider the comparison function,

$$
v(y)=1-y_{N}^{2}, \quad 0 \leq y_{N} \leq \lambda,
$$

and let

$$
\bar{w}_{\lambda}(y)=\frac{w_{\lambda}(y)}{v(y)}, \quad \text { i.e., } w_{\lambda}(y)=\bar{w}_{\lambda}(y) v(y)
$$

Thus, $\bar{w}_{\lambda}$ satisfies

$$
\left\{\begin{array}{l}
\Delta \bar{w}_{\lambda}(y)+2 \frac{\nabla v(y)}{v(y)} \cdot \nabla \bar{w}_{\lambda}(y)+\left(C_{1}(y)+C_{2}(y)-\frac{4}{v(y)}\right) \bar{w}_{\lambda}(y) \leq 0, \\
\bar{w}_{\lambda}\left(y^{\prime}, 0\right)>0 \quad \text { and } \quad \bar{w}_{\lambda}\left(y^{\prime}, \lambda\right)=0 .
\end{array}\right.
$$

Choose $\lambda$ small such that

$$
\frac{N+2}{N-2} u^{\frac{4}{N-2}}(y) \leq 2, \quad 0 \leq y_{N} \leq \lambda
$$

Now suppose the set $\left\{y \mid w_{\lambda}(y)<0\right\} \neq \emptyset$.

Because $\bar{w}_{\lambda} \geq 0$ on $\partial \Sigma_{\lambda}$ and $\lim _{|y| \rightarrow+\infty} \bar{w}_{\lambda}(y)=0$, it is easy to see the minimum of $\bar{w}_{\lambda}$ can be achieved. Let $\bar{y} \in \Sigma_{\lambda}$ such that

$$
\bar{w}_{\lambda}(\bar{y})=\inf _{y \in \Sigma_{\lambda}} \bar{w}_{\lambda}(y)<0
$$

Since $\bar{w}_{\lambda}(\bar{y})<0$

$$
C(\bar{y}) \leq \frac{N+2}{N-2} u^{\frac{4}{N-2}}(\bar{y}) \leq 2 .
$$

By applying the maximum principle, (2.14) yields

$$
0<\left(C_{1}(\bar{y})+C_{2}(\bar{y})-\frac{4}{v(\bar{y})}\right) \bar{w}_{\lambda}(\bar{y}) \leq 0
$$

which is a contradiction. Hence, $w_{\lambda}(y)>0 \forall y \in \Sigma_{\lambda}$.

Let

$$
\bar{\lambda}=\sup \left\{\lambda \mid w_{\mu}(y)>0 \forall y \in \Sigma_{\mu}, 0<\mu \leq \lambda\right\} .
$$

We claim $\bar{\lambda}=+\infty$. Otherwise, we have

$$
\left\{\begin{array}{l}
w_{\bar{\lambda}}(y)>0 \quad \forall y \in \Sigma_{\bar{\lambda}} \\
\frac{\partial w_{\bar{\lambda}}}{\partial y_{N}}\left(y^{\prime}, \lambda\right)<0
\end{array}\right.
$$

by the strong maximum principle and Hopf boundary point lemma. By the definition of $\bar{\lambda}$, there are $\lambda_{j} \downarrow \bar{\lambda}$ such that $\left\{y \mid w_{\lambda_{j}(y)}<0, y \in \Sigma_{\lambda_{j}}\right\} \neq \emptyset$. Set

$$
w_{\lambda_{j}}(y)=\bar{w}_{\lambda_{j}}(y) v(y),
$$

where

$$
v(y)=(\bar{\lambda}+1)^{2}-y_{N}^{2}, y \in \Sigma_{\lambda_{j}} .
$$

Then $\bar{w}_{\lambda_{j}}$ satisfies

$$
\begin{aligned}
\Delta \bar{w}_{\lambda_{j}}(y) & +2 \nabla \log v(y) \cdot \nabla \bar{w}_{\lambda_{j}}(y) \\
& +\left(C_{1}(y)+C_{2}(y)-\frac{4}{v(y)}\right) \bar{w}_{\lambda_{j}} \leq 0
\end{aligned}
$$


Suppose $\bar{w}_{\lambda_{j}}\left(\bar{y}_{j}\right)=\inf _{y \in \Sigma_{\lambda_{j}}} \bar{w}_{\lambda_{j}}(y)<0$. By (2.15), we have $\left|\bar{y}_{j}\right| \rightarrow+\infty$. Note that

$$
C_{2}\left(\bar{y}_{j}\right) \leq \frac{N+2}{N-2} u^{\frac{4}{N-2}}\left(\bar{y}_{j}\right) \rightarrow 0 \quad \text { as } j \rightarrow+\infty .
$$

Again, by the maximum principle, (2.16) yields a contradiction. Therefore, Step 2 is proved. Obviously, the conclusion of Lemma 2.1 follows immediately from Step 2.

After the proof $\frac{\partial u}{\partial x_{N}}>0$, it is clear from the last step in the proof of Theorem [1.3, that the conclusion of Theorem 2.2 holds.

Remark 2.3. If $P_{i}=P_{j} \forall i \neq j$, then the proof of Lemma 2.1 still holds. Hence, in this case, the boundedness assumption is not necessary for the conclusion of Theorem 2.2

\section{Existence of Entire Solutions}

In this section, we will give a proof of Theorem[1.2 To prove Theorem 1.2, we choose a convex domain $\Omega$ with $0 \in \partial \Omega$ and consider the following equation. For any small $\varepsilon>0$

$$
\left\{\begin{array}{l}
\Delta u+\lambda \frac{u^{p_{1}(\varepsilon)}}{|x|^{s_{1}}}+\frac{u^{p_{2}(\varepsilon)}}{|x|^{s_{2}}}=0 \text { in } \Omega, \\
u(x)>0 \text { in } \Omega \text { and } u(x)=0 \text { on } \partial \Omega,
\end{array}\right.
$$

where $p_{1}(\varepsilon)=2^{*}\left(s_{1}\right)-1-\varepsilon$ and $p_{2}(\varepsilon)=2^{*}\left(s_{2}\right)-1-\left(\frac{2-s_{2}}{2-s_{1}}\right) \varepsilon$.

For $\varepsilon>0$, we let

$$
\Phi_{\varepsilon}(u):=\frac{1}{2} \int_{\Omega}|\nabla u|^{2} d x-\frac{\lambda}{p_{1}(\varepsilon)+1} \int_{\Omega} \frac{\left(u^{+}\right)^{p_{1}(\varepsilon)+1}}{|x|^{s_{1}}} d x-\frac{1}{p_{2}(\varepsilon)+1} \int_{\Omega} \frac{\left(u^{+}\right)^{p_{2}(\varepsilon)+1}}{|x|^{s_{2}}} d x
$$

for $u \in H_{0}^{1}(\Omega)$ and

$$
c_{\varepsilon}^{*}=\inf _{P \in \mathcal{P}} \max _{w \in P} \Phi_{\varepsilon}(w),
$$

where $\mathcal{P}$ is the class of all continuous paths in $H_{0}^{1}(\Omega)$ connecting 0 and some $u_{0}$ such that $\Phi_{\varepsilon}\left(u_{0}\right) \leq 0$. It is easy to see that $c_{\varepsilon}^{*} \leq C$ for some constant $C$ independent of $\varepsilon$. Since for $\varepsilon>0, \Phi_{\varepsilon}$ satisfies the P-S condition, it is known that $c_{\varepsilon}^{*}$ is a critical point of $\Phi_{\varepsilon}$, i.e., there exists a solution $u_{\varepsilon} \in H_{0}^{1}(\Omega)$ with $\Phi_{\varepsilon}\left(u_{\varepsilon}\right)=c_{\varepsilon}^{*}$. Thus,

$$
\left\{\begin{array}{l}
\frac{1}{2} \int_{\Omega}\left|\nabla u_{\varepsilon}\right|^{2} d x-\frac{\lambda}{p_{1}(\varepsilon)+1} \int_{\Omega} \frac{u_{\varepsilon}^{p_{1}(\varepsilon)+1}}{|x|^{s_{1}}} d x-\frac{1}{p_{2}(\varepsilon)+1} \int_{\Omega} \frac{u_{\varepsilon}^{p_{2}(\varepsilon)+1}}{|x|^{s_{2}}} d x=c_{\varepsilon}^{*} \\
\int_{\Omega}\left|\nabla u_{\varepsilon}\right|^{2} d x-\lambda \int_{\Omega} \frac{u_{\varepsilon}^{p_{\varepsilon}(\varepsilon)+1}}{|x|^{s_{1}}} d x-\int_{\Omega} \frac{u_{\varepsilon}^{p_{2}(\varepsilon)+1}}{|x|^{s_{2}}} d x=0 .
\end{array}\right.
$$

From (3.2), we have

$$
\left(\frac{1}{2}-\frac{1}{p_{1}(\varepsilon)+1}\right) \int_{\Omega}\left|\nabla u_{\varepsilon}\right|^{2} d x+\left(\frac{1}{p_{1}(\varepsilon)+1}-\frac{1}{p_{2}(\varepsilon)+1}\right) \int_{\Omega} \frac{u_{\varepsilon}^{p_{2}(\varepsilon)+1}}{|x|^{s_{2}}} d x=c_{\varepsilon}^{*} .
$$

By noting both of $\frac{1}{2}-\frac{1}{p_{1}(\varepsilon)+1}$ and $\frac{1}{p_{1}(\varepsilon)+1}-\frac{1}{p_{2}(\varepsilon)+1}$ are positive, we have

$$
\int_{\Omega}\left|\nabla u_{\varepsilon}\right|^{2} d x+\int_{\Omega} \frac{u_{\varepsilon}^{p_{1}(\varepsilon)+1}}{|x|^{s_{1}}} d x+\int_{\Omega} \frac{u_{\varepsilon}^{p_{2}(\varepsilon)+1}}{|x|^{s_{2}}} d x \leq C<+\infty .
$$


Therefore, by passing to a subsequence if necessary, we might assume $u_{\varepsilon} \rightarrow u$ in $H_{0}^{1}(\Omega)$ as $\varepsilon \rightarrow 0$. If $u \not \equiv 0$, then $u$ is a solution of

$$
\left\{\begin{array}{l}
\Delta u+\lambda \frac{u^{p_{1}}}{|x|^{s_{1}}}+\frac{u^{p_{2}}}{|x|^{s_{2}}}=0 \text { in } \Omega, \\
u=0 \text { on } \partial \Omega .
\end{array}\right.
$$

However, the standard Pohozaev indentity yields that equation (3.4) has no positive solutions because both of $p_{1}$ and $p_{2}$ are critical exponents. Thus $u \equiv 0$ and $u_{\varepsilon}(x)$ must blow up as $\varepsilon \rightarrow 0$.

Before poceeding further, we will briefly discuss the regularity of $u$ at 0 . Because $s_{1}<2$, we can prove that

$$
\left\{\begin{array}{l}
u \in C^{2}(\bar{\Omega}) \quad \text { if } s_{1}<1+\frac{2}{n}, \\
u \in C^{1, \beta}(\bar{\Omega}) \quad \text { for all } 0<\beta<1 \text { if } s_{1}=1+\frac{2}{n}, \\
u \in C^{1, \beta}(\bar{\Omega}) \quad \text { for all } 0<\beta<\frac{n(2-s)}{n-2} \text { if } s_{1}>1+\frac{2}{n},
\end{array}\right.
$$

see [17.

Let

$$
u_{\varepsilon}\left(x_{\varepsilon}\right)=\max _{\bar{\Omega}} u_{\varepsilon}(x)=m_{\varepsilon} \text { and } k_{\varepsilon}=m_{\varepsilon}^{-\frac{p_{2}(\varepsilon)-1}{2-s_{2}}} .
$$

By direct computations, we have

$$
k_{\varepsilon}=m_{\varepsilon}^{-\frac{2}{N-2}+\frac{\varepsilon}{2-s_{1}}} .
$$

First, we claim

$$
\left|x_{\varepsilon}\right|=O\left(k_{\varepsilon}\right) .
$$

Suppose not. By passing to a subsequence if necessary, we may assume

$$
\lim _{\varepsilon \rightarrow 0} \frac{\left|x_{\varepsilon}\right|}{k_{\varepsilon}}=+\infty
$$

By scaling, we set

$$
\tilde{v}_{\varepsilon}(y)=\frac{u_{\varepsilon}\left(x_{\varepsilon}+r_{\varepsilon} y\right)}{m_{\varepsilon}} \text { in } \Omega_{\varepsilon}
$$

where

$$
\Omega_{\varepsilon}=\left\{y \in \mathbb{R}^{N} \mid x_{\varepsilon}+r_{\varepsilon} y \in \Omega\right\}, \quad \text { and } \quad r_{\varepsilon}=\left|x_{\varepsilon}\right|^{\frac{s_{2}}{2}} k_{\varepsilon}^{\frac{2-s_{2}}{2}}=\left(\frac{\left|x_{\varepsilon}\right|}{k_{\varepsilon}}\right)^{\frac{s_{2}}{2}} k_{\varepsilon} .
$$

By equation (3.1), $\tilde{v}_{\varepsilon}(y)$ satisfies

$$
\left\{\begin{array}{l}
\Delta \tilde{v}_{\varepsilon}+\lambda\left(\frac{k_{\varepsilon}}{\left|x_{\varepsilon}\right|}\right)^{s_{1}-s_{2}} \frac{\tilde{v}_{\varepsilon}^{p_{1}(\varepsilon)}}{\left|y_{\varepsilon}+\frac{r_{\varepsilon}}{\mid x_{\varepsilon}} y\right|^{s_{1}}}+\frac{\tilde{v}_{\varepsilon}^{p_{2}}(\varepsilon)}{\left|y_{\varepsilon}+\frac{r_{\varepsilon}}{\left|x_{\varepsilon}\right|} y\right|^{s_{2}}}=0 \quad \text { in } \Omega_{\varepsilon} \\
y_{\varepsilon}=\frac{x_{\varepsilon}}{\left|x_{\varepsilon}\right|} \quad \text { and } \quad \tilde{v}_{\varepsilon}(y) \leq \tilde{v}_{\varepsilon}(0)=1
\end{array}\right.
$$

Let $\Omega_{\varepsilon} \rightarrow H$ as $\varepsilon \rightarrow 0$, where either $H=\mathbb{R}^{N}$ or $H$ is a closed half space of $\mathbb{R}^{N}$. Note $\left(\frac{k_{\varepsilon}}{x_{\varepsilon}}\right)^{s_{1}-s_{2}}$ and $\frac{r_{\varepsilon}}{\left|x_{\varepsilon}\right|}=\left(\frac{k_{\varepsilon}}{\left|x_{\varepsilon}\right|}\right)^{\frac{2-s_{2}}{2}}$ tend to 0 as $\varepsilon \rightarrow 0$. Then by applying elliptic estimates, $\tilde{v}_{\varepsilon}$ converges to $\tilde{v}$ in $C_{l o c}^{2}(H)$, where

$$
\Delta \tilde{v}+\tilde{v}^{p_{2}}=0 \quad \text { in } H
$$


If $H$ is a half space of $\mathbb{R}^{N}$, then $v$ also satisfies $v=0$ on $\partial H$. Since $p_{2}=\frac{2\left(N-s_{2}\right)}{N-2}-1<\frac{N+2}{N-2}$, $v(y) \equiv 0$ in $H$ no matter $H$ is $\mathbb{R}^{N}$ or a half space. But it yields a contradiction to $v(0)=1$. Thus, the claim is proved.

After (3.6) is established, we set

$$
v_{\varepsilon}(y)=m_{\varepsilon}^{-1} u_{\varepsilon}\left(x_{\varepsilon}+k_{\varepsilon} y\right) .
$$

Then $v_{\varepsilon}(y)$ satisfies

$$
\Delta v_{\varepsilon}+\lambda \frac{v_{\varepsilon}^{p_{1}(\varepsilon)}}{\left|\frac{x_{\varepsilon}}{k_{\varepsilon}}+y\right|^{s_{1}}}+\frac{v_{\varepsilon}^{p_{2}(\varepsilon)}}{\left|\frac{x_{\varepsilon}}{k_{\varepsilon}}+y\right|^{s_{2}}}=0 \text { in } \Omega_{\varepsilon},
$$

where $\Omega_{\varepsilon}=\left\{y \in \mathbb{R}^{N} \mid x_{\varepsilon}+k_{\varepsilon} y \in \Omega\right\}$. Since $\frac{x_{\varepsilon}}{k_{\varepsilon}}$ is bounded, without loss of generality, we may assume $\frac{x_{\varepsilon}}{k_{\varepsilon}} \rightarrow y_{0}$. Therefore, $\Omega_{\varepsilon} \rightarrow H$ as $\varepsilon \rightarrow 0$, where $H$ is a half spae of $\mathbb{R}^{N}$ with $-y_{0} \in \partial H$ and by the elliptic estimates, $v_{\varepsilon}(y) \rightarrow v(y)$ in $C_{l o c}^{2}(H)$. Clearly, $v$ satisfies

$$
\left\{\begin{array}{l}
\Delta v+\lambda \frac{v^{p_{1}}}{\left|y_{0}+y\right|^{s_{1}}}+\frac{v^{p_{2}}}{\left|y_{0}+y\right|^{s_{2}}}=0 \text { in } H, \\
v=0 \text { on } \partial H .
\end{array}\right.
$$

Since $v(0)=1$, we have $y_{0} \neq 0$. By a linear transformation of $y, H$ can be map onto $\mathbb{R}_{+}^{N}$ and $v$ is an entire solution of equation (1.1) with $\Omega=\mathbb{R}_{+}^{N}$. This completes the proof of the existence part of Theorem 1.2

Remark. Suppose $v$ is a positive entire soltion of (1.1). Then the Kelvin transformation $\hat{v}(y)=|y|^{2-n} v\left(\frac{y}{|y|^{2}}\right)$ is also a positive entire solution. By the regularity (3.5), $|\hat{v}(y)| \leq C|y|$ for $|y|<1$. Thus,

$$
|v(y)| \leq C|y|^{1-n} \quad \text { for }|y| \geq 1 .
$$

By the standard gradient estimate, we have

$$
|\nabla v(y)| \leq|y|^{-n} \quad \text { for }|y| \geq 1 \text {. }
$$

By using the well-known method of moving sphere, it can be proved that after a suitable scaling, $v(y)=\hat{v}(y)$. Since the argument is standard now, the proof is omitted here.

Corollary 3.1. There exists an entire solution $v$ of equation (1.1) with $\Omega=\mathbb{R}_{+}^{N}$ such that the critical value $\Phi(v)=\inf \{\Phi(u) \mid u$ is an entire solution of (1.1) $\}$.

Proof. We first note that

$$
\{\Phi(u) \mid \mathrm{u} \text { is a positive entire solution of (1.1) }\} \neq \emptyset
$$

because

$$
\begin{aligned}
\int_{\mathbb{R}_{+}^{N}}|\nabla u|^{2} d y & =\lambda \int_{\mathbb{R}_{+}^{N}} \frac{u^{2^{*}\left(s_{1}\right)}}{|x|^{s_{1}}} d y+\int_{\mathbb{R}_{+}^{N}} \frac{u^{2^{*}\left(s_{2}\right)}}{|x|^{s_{2}}} d y \\
& \leq C\left[\left(\int_{\mathbb{R}_{+}^{N}}|\nabla u|^{2} d y\right)^{\frac{2^{*}\left(s_{1}\right)}{2}}+\left(\int_{\mathbb{R}_{+}^{N}}|\nabla u|^{2} d y\right)^{\frac{2^{*}\left(s_{2}\right)}{2}}\right]
\end{aligned}
$$

implies $\|\nabla u\| \geq c_{0}$ for some constant $c_{0}>0$. 
Suppose $v_{j}$ is a sequence of positive entire solutions of

$$
\left\{\begin{array}{l}
\Delta v_{j}+\lambda \frac{v_{j}^{p_{1}}}{|y|^{s_{1}}}+\frac{v_{j}^{p_{2}}}{|y|^{s_{2}}}=0 \text { in } \mathbb{R}_{+}^{N}, \\
v_{j}=0 \text { on } \partial \mathbb{R}_{+}^{N}
\end{array}\right.
$$

such that $\Phi\left(v_{j}\right) \downarrow \inf \{\Phi(u) \mid u$ is an entire solution of (1.1) $\}$. By the remark above, we can assume $\hat{v}_{j}(y)=v_{j}(y)$. By (3.2) again, $\left\|\nabla v_{j}\right\|_{L^{2}\left(\mathbb{R}_{+}^{N}\right)} \leq C$ for some constant $C>0$. Let $v_{j} \rightarrow v$ in $H_{0}^{1}\left(\mathbb{R}_{+}^{n}\right)$. If $v \neq 0$, then

$$
\begin{aligned}
\lim _{j \rightarrow+\infty} \Phi\left(v_{j}\right) & =\left(\frac{1}{2}-\frac{1}{p_{1}+1}\right) \lim _{j \rightarrow \infty} \int_{\mathbb{R}_{+}^{N}}\left|\nabla v_{j}\right|^{2} d y+\left(\frac{1}{p_{1}+1}-\frac{1}{p_{2}+1}\right) \lim _{j \rightarrow+\infty} \int_{\mathbb{R}_{+}^{N}} \frac{v_{j}^{p_{2}+1}}{|y|^{s_{2}}} d y \\
& \geq\left(\frac{1}{2}-\frac{1}{p_{1}+1}\right) \int_{\mathbb{R}_{+}^{N}}|\nabla v|^{2} d y+\left(\frac{1}{p_{1}+1}-\frac{1}{p_{2}+1}\right) \int_{\mathbb{R}_{+}^{N}} \frac{v^{p_{2}+1}}{|y|^{s_{2}}} d y=\Phi(v) .
\end{aligned}
$$

Then it yields the conclusion of Corollary 3.1

If $v_{j} \rightarrow 0$, then $\max _{|y| \leq 2} v_{j}(y) \rightarrow+\infty$, because $\hat{v}_{j}(y)=v_{j}(y)$ implies

$$
\begin{aligned}
\frac{1}{2} \Phi\left(v_{j}\right) & =\frac{1}{2} \int_{B_{1}}\left|\nabla v_{j}\right|^{2} d y-\frac{\lambda}{p_{1}+1} \int_{B_{1}} \frac{v_{j}^{p_{1}+1}}{|y|^{s_{1}}} d y-\frac{1}{p_{2}+1} \int_{B_{2}} \frac{v_{j}^{p_{2}+1}}{|y|^{s_{2}}} d y \\
& =\left(\frac{1}{2}-\frac{1}{p_{1}+1}\right) \int_{B_{1}}\left|\nabla v_{j}\right|^{2} d y+\left(\frac{1}{p_{1}+1}-\frac{1}{p_{2}+1}\right) \int_{B_{2}} \frac{v_{j}^{p_{2}+1}}{|y|^{s_{2}}} d y .
\end{aligned}
$$

By the proof of Theorem 1.2 we see that $v_{j}$ blows up at $y=0$ and the scaling $w_{j}(y)$ :

$$
w_{j}(y)=\frac{v_{j}\left(x_{j}+k_{j} y\right)}{v_{j}\left(x_{j}\right)} \rightarrow w
$$

where $v_{j}\left(x_{j}\right)=\max _{|y| \leq 2} v_{j}(y) \rightarrow \infty, k_{j}=m_{j}^{-\frac{2}{N-2}}$, and $w$ is also a positive entire solution of equation (1.1). Thus,

$$
\lim _{j \rightarrow+\infty} \frac{1}{2} \Phi\left(v_{j}\right) \geq\left(\frac{1}{2}-\frac{1}{p_{1}+1}\right) \int_{\mathbb{R}_{+}^{N}}|\nabla w|^{2} d y+\left(\frac{1}{p_{1}+1}-\frac{1}{p_{2}+1}\right) \int_{\mathbb{R}_{+}^{N}} \frac{w^{p_{2}+1}}{|y|^{s_{2}}} d y=\Phi(w),
$$

which yields

$$
\inf \{\Phi(u) \mid u \text { is an entire solution of equation (1.1) }=0\} \text {, }
$$

a contradiction. Hence, $v_{j} \neq 0$, and Corollary3.1 is proved.

\section{Proof of Theorem 1.1}

Let $v(y)$ be a least-energy solution of

$$
\begin{cases}\Delta v+\lambda \frac{v^{p_{1}}}{\mid y^{s_{1}}}+\frac{v^{p_{2}}}{||^{s_{2}}}=0 & \text { in } \mathbb{R}_{+}^{N}, \\ v(y)>0 \text { in } \mathbb{R}_{+}^{N} \text { and } & v(y)=0 \text { on } \partial \mathbb{R}_{+}^{N},\end{cases}
$$

and

$$
c_{1}=\Phi(v)=\frac{1}{2} \int_{\mathbb{R}_{+}^{N}}|\nabla v|^{2} d y-\frac{\lambda}{2^{*}\left(s_{1}\right)} \int_{\mathbb{R}_{+}^{N}} \frac{v^{2^{*}\left(s_{1}\right)}}{|y|^{s_{1}}} d y-\frac{1}{2^{*}\left(s_{2}\right)} \int_{\mathbb{R}_{+}^{N}} \frac{v^{2^{*}\left(s_{2}\right)}}{|y|^{s_{2}}} d y .
$$


Lemma 4.1. Suppose that $\Omega$ is a bounded smooth domain in $\mathbb{R}^{N}$ with $0 \in \partial \Omega$. If $H(0)<0$, then there exists a nonnegative function $v_{0} \in H_{0}^{1}(\Omega) \backslash\{0\}$ such that

$$
\max _{t \geq 0} \Phi\left(t v_{0}\right)<c_{1}
$$

Proof. Without loss of generality, we may assume that in a neighborhood of $0, \partial \Omega$ can be represented by $x_{n}=\varphi\left(x^{\prime}\right)$, where $x^{\prime}=\left(x_{1}, \cdots, x_{N-1}\right), \varphi(0)=0, \nabla^{\prime} \varphi(0)=0, \nabla^{\prime}=$ $\left(\partial_{1}, \cdots, \partial_{N-1}\right)$, and the outer normal of $\partial \Omega$ at 0 is $-e_{N}=(0, \cdots, 0,-1)$. Define

$$
\phi(x):=\left(x^{\prime}, x_{N}-\varphi\left(x^{\prime}\right)\right)
$$

We choose a small positive number $r_{0}$ so that there exist neighborhoods of $0, U$ and $\tilde{U}$, such that $\phi(U)=B_{r_{0}}(0), \phi(U \cap \Omega)=B_{r_{0}}^{+}(0), \phi(\tilde{U})=B_{\frac{r_{0}}{2}}(0)$ and $\phi(\tilde{U} \cap \Omega)=B_{\frac{r_{0}}{2}}^{+}(0)$. Here, we adopt the notation:

$$
B_{r_{0}}^{+}(0)=B_{r_{0}} \cap \mathbb{R}_{+}^{N} \text { for } r_{0}>0 .
$$

Let $\eta \in C_{0}^{\infty}(U)$ be a positive cut-off function with $\eta \equiv 1$ in $\tilde{U}$. Set

$$
u_{\varepsilon}(x):=\eta(x) v_{\varepsilon}(x):=\eta(x) \varepsilon^{-\frac{N-2}{2}} v\left(\frac{\phi(x)}{\varepsilon}\right) \quad \text { for } x \in \Omega .
$$

For $t \geq 0$, we have

$$
\Phi\left(t u_{\varepsilon}\right)=\frac{t^{2}}{2} \int_{\Omega}\left|\nabla u_{\varepsilon}\right|^{2} d x-\frac{\lambda t^{2^{*}\left(s_{1}\right)}}{2^{*}\left(s_{1}\right)} \int_{\Omega} \frac{u_{\varepsilon}^{2^{*}\left(s_{1}\right)}}{|x|^{s_{1}}} d x-\frac{t^{2^{*}\left(s_{2}\right)}}{2^{*}\left(s_{2}\right)} \int_{\Omega} \frac{u_{\varepsilon}^{2^{*}\left(s_{2}\right)}}{|x|^{s_{2}}} d x \quad \text { for } u \in H_{0}^{1}(\Omega) .
$$

In what follows, we estimate each integral on the right-hand side of (4.3). Basically, the computation will be similar to Lemma 2.2 in [11. For the sake of completeness, we will sketch the proof here. We refer the readers to [11] for details of computation.

By the change of the variable $\frac{\phi(x)}{\varepsilon}=y$, we get

$$
\begin{aligned}
& \int_{\Omega}\left|\nabla u_{\varepsilon}\right|^{2} d x=\int_{U \cap \Omega} \eta^{2}\left|\nabla v_{\varepsilon}\right|^{2} d x-\int_{U \cap \Omega} \eta(\Delta \eta) v_{\varepsilon}^{2} d x \\
& =\int_{\mathbb{R}_{+}^{N}}|\nabla v(y)|^{2} d y-2 \int_{B_{\frac{r_{0}}{\varepsilon}}^{+}} \eta\left(\phi^{-1}(\varepsilon y)\right)^{2} \partial_{N} v(y) \nabla^{\prime} v(y) \cdot\left(\nabla^{\prime} \varphi\right)\left(\varepsilon y^{\prime}\right) d y+O\left(\varepsilon^{2}\right) .
\end{aligned}
$$

By using integration by parts and equation (4.1), the second term can be estimated as the following.

$$
\begin{aligned}
& -2 \int_{B_{\frac{r_{0}}{\varepsilon}}^{+}} \eta\left(\phi^{-1}(\varepsilon y)\right)^{2} \partial_{N} v(y) \nabla^{\prime} v(y) \cdot\left(\nabla^{\prime} \varphi\right)\left(\varepsilon y^{\prime}\right) d y \\
& =\frac{2}{\varepsilon} \int_{B_{\frac{r_{0}}{\varepsilon}}^{+}} \eta\left(\phi^{-1}(\varepsilon y)\right)^{2} \partial_{N} v(y) \sum_{i=1}^{N-1} \partial_{i i} v(y) \varphi\left(\varepsilon y^{\prime}\right)+O\left(\varepsilon^{2}\right) \\
& =-\frac{1}{\varepsilon} \int_{B_{\frac{r_{0}}{\varepsilon}}^{+}} \eta\left(\phi^{-1}(\varepsilon y)\right)^{2} \partial_{N}\left[\left(\partial_{N} v(y)\right)^{2}\right] \varphi\left(\varepsilon y^{\prime}\right) d y \\
& -\frac{2 \lambda}{2^{*}\left(s_{1}\right) \varepsilon} \int_{B_{\frac{r_{0}}{\varepsilon}}^{+}} \eta\left(\phi^{-1}(\varepsilon y)\right)^{2} \frac{\partial_{N}\left[v(y)^{2^{*}\left(s_{1}\right)}\right]}{|y|^{s_{1}}} \varphi\left(\varepsilon y^{\prime}\right) d y
\end{aligned}
$$




$$
\begin{aligned}
& -\frac{2}{2^{*}\left(s_{2}\right) \varepsilon} \int_{B_{\frac{r_{0}}{\varepsilon}}^{+}} \eta\left(\phi^{-1}(\varepsilon y)\right)^{2} \frac{\partial_{N}\left[v(y)^{2^{*}\left(s_{2}\right)}\right]}{|y|^{s_{2}}} \varphi\left(\varepsilon y^{\prime}\right) d y+O\left(\varepsilon^{2}\right) \\
& =: I_{1}+I_{2}+I_{3}+O\left(\varepsilon^{2}\right) .
\end{aligned}
$$

Since $\partial \Omega$ is $C^{2}$ at $0, \varphi$ can be expanded as

$$
\varphi\left(y^{\prime}\right)=\sum_{i=1}^{N-1} \alpha_{i} y_{i}^{2}+o(1)\left|y^{\prime}\right|^{2} .
$$

Hence,

$$
\begin{aligned}
I_{1} & =\frac{1}{\varepsilon} \int_{B_{\frac{r_{0}}{\varepsilon}}^{+} \cap \mathbb{R}_{+}^{N}} \eta\left(\phi^{-1}\left(\varepsilon y^{\prime}\right)\right)^{2} \partial_{N} v\left(y^{\prime}, 0\right) \varphi\left(\varepsilon y^{\prime}\right) d y^{\prime} \\
& =\varepsilon \sum_{i=1}^{N-1} \alpha_{i} \int_{\mathbb{R}^{N-1}}\left(\partial_{N} v\left(y^{\prime}, 0\right)\right)^{2} y_{i}^{2} d y^{\prime}(1+o(1))+O\left(\varepsilon^{2}\right)=K_{1} H(0) \varepsilon(1+o(1))+O\left(\varepsilon^{2}\right),
\end{aligned}
$$

where

$$
\begin{gathered}
K_{1}=\int_{\mathbb{R}^{N-1}}\left|\partial_{N} v\left(y^{\prime}, 0\right)\right|^{2}\left|y^{\prime}\right|^{2} d y^{\prime}, \\
I_{2}=-\frac{2 \lambda s_{1}}{2^{*}\left(s_{1}\right) \varepsilon} \int_{B_{\frac{r_{0}}{\varepsilon}}^{+}} \eta\left(\phi^{-1}(\varepsilon y)\right)^{2} \frac{v(y)^{2^{*}\left(s_{1}\right)} y_{N}}{|y|^{2+s_{1}}} \varphi\left(\varepsilon y^{\prime}\right) d y \\
=-K_{2} H(0)(1+o(1)) \varepsilon+O\left(\varepsilon^{2}\right),
\end{gathered}
$$

where

$$
K_{2}=\frac{2 \lambda s_{1}}{2^{*}\left(s_{1}\right)} \int_{\mathbb{R}_{+}^{N}} \frac{v(y)^{2^{*}\left(s_{1}\right)}\left|y^{\prime}\right|^{2} y_{N}}{|y|^{2+s_{1}}} d y
$$

and

$$
\begin{aligned}
I_{3} & =-\frac{2 s_{2}}{2^{*}\left(s_{2}\right) \varepsilon} \int_{B_{\frac{r_{0}}{\varepsilon}}^{+}} \eta\left(\phi^{-1}(\varepsilon y)\right)^{2} \frac{v(y)^{2^{*}\left(s_{2}\right)}\left|y^{\prime}\right|^{2} y_{N}}{|y|^{2+s_{2}}} \varphi\left(\varepsilon y^{\prime}\right) d y \\
& =-K_{3} H(0) \varepsilon(1+o(1))+O\left(\varepsilon^{2}\right),
\end{aligned}
$$

where

$$
K_{3}=\frac{2 s_{2}}{2^{*}\left(s_{2}\right)} \int_{\mathbb{R}_{+}^{N}} \frac{v(y)^{2^{*}\left(s_{2}\right)}\left|y^{\prime}\right|^{2} y_{N}}{|y|^{2+s_{2}}} d y .
$$

By (3.8) and (3.9), $K_{i}, i=1,2,3$, are finite. Therefore, we have

$$
\int_{\Omega}\left|\nabla u_{\varepsilon}\right|^{2} d x=\int_{\mathbb{R}_{+}^{N}}|\nabla v|^{2} d y+\varepsilon H(0)\left(K_{1}-K_{2}-K_{3}\right)(1+o(1))+O\left(\varepsilon^{2}\right),
$$

and similarly, we have

$$
\lambda \int_{\Omega} \frac{u_{\varepsilon}^{2^{*}\left(s_{1}\right)}}{|x|^{s_{1}}} d x=\lambda \int_{\mathbb{R}_{+}^{N}} \frac{v^{2^{*}\left(s_{1}\right)}}{|y|^{s_{1}}} d y-\frac{s \lambda}{\varepsilon} \int_{B_{\frac{r_{0} / 2}{\varepsilon}}^{+}} \frac{v(y)^{2^{*}\left(s_{1}\right)} y_{N} \varphi\left(\varepsilon y^{\prime}\right)}{|y|^{2+s_{1}}} d y+O\left(\varepsilon^{2}\right)
$$




$$
=\lambda \int_{\mathbb{R}_{+}^{N}} \frac{v^{2^{*}\left(s_{1}\right)}}{|y|^{s_{1}}} d y-2^{*}\left(s_{1}\right) K_{2} H(0) \varepsilon(1+o(1))+O\left(\varepsilon^{2}\right)
$$

and

$$
\int_{\Omega} \frac{u_{\varepsilon}^{2^{*}\left(s_{2}\right)}}{|x|^{s_{2}}} d x=\int_{\mathbb{R}_{+}^{N}} \frac{v^{2^{*}\left(s_{2}\right)}}{|y|^{s_{2}}} d y-2^{*}\left(s_{2}\right) K_{3} H(0) \varepsilon(1+o(1))+O\left(\varepsilon^{2}\right) .
$$

Therfore,

$$
\begin{aligned}
\Phi\left(t u_{\varepsilon}\right) & =\frac{t^{2}}{2} \int_{\mathbb{R}_{+}^{N}}|\nabla v|^{2} d y-\frac{\lambda t^{2^{*}\left(s_{1}\right)}}{2^{*}\left(s_{1}\right)} \int_{\mathbb{R}_{+}^{N}} \frac{v^{2^{*}\left(s_{1}\right)}}{|y|^{s_{1}}} d y-\frac{t^{2^{*}\left(s_{2}\right)}}{2^{*}\left(s_{2}\right)} \int_{\mathbb{R}_{+}^{N}} \frac{v^{2^{*}\left(s_{2}\right)}}{|y|^{s_{2}}} d y \\
& +\frac{t^{2}}{2}\left(\varepsilon H(0)\left(K_{1}-K_{2}-K_{3}\right)+o(1)\right)+\frac{t^{2^{*}\left(s_{1}\right)}}{2} K_{2} H(0)(1+o(1)) \\
& +\frac{t^{2^{*}\left(s_{2}\right)}}{2} K_{3} H(0)(1+o(1)) \varepsilon+O\left(\varepsilon^{2}\right)=: f_{1}(t)+f_{2}(t)+O\left(\varepsilon^{2}\right),
\end{aligned}
$$

where

$$
f_{1}(t)=\frac{t^{2}}{2} \int_{\mathbb{R}_{+}^{N}}|\nabla v|^{2} d y-\frac{\lambda t^{2^{*}\left(s_{1}\right)}}{2^{*}\left(s_{1}\right)} \int_{\mathbb{R}_{+}^{N}} \frac{v^{2^{*}\left(s_{1}\right)}}{|y|^{s_{1}}} d y-\frac{t^{2^{*}\left(s_{2}\right)}}{2^{*}\left(s_{2}\right)} \int_{\mathbb{R}_{+}^{N}} \frac{v^{2^{*}\left(s_{1}\right)}}{|y|^{s_{1}}} d y .
$$

Since $2^{*}\left(s_{2}\right)>2^{*}\left(s_{1}\right), \Phi\left(t u_{\varepsilon}\right)$ has the unique maximum. Note that

$$
\max _{t \geq 0} f_{1}(t)=f_{1}(1)=c_{1} .
$$

Hence, the maximum of $\Phi\left(t u_{\varepsilon}\right)$ occurs at $t_{\varepsilon}=1+o(1)$. By noting that

$$
f_{2}(t)=\varepsilon H(0)\left[\frac{t^{2}}{2}\left(K_{1}-K_{2}-K_{3}+o(1)\right)+\frac{t^{2^{*}\left(s_{1}\right)}}{2} K_{2}(1+o(1))+\frac{t^{2^{*}\left(s_{2}\right)}}{2} K_{3}(1+o(1))\right],
$$

and $f_{2}(1)=\varepsilon H(0) K_{1}(1+o(1))<0$. Hence, we have

$$
\max _{t \geq 0} \Phi\left(t u_{\varepsilon}\right)=\Phi\left(t_{\varepsilon} u_{\varepsilon}\right) \leq f_{1}\left(t_{\varepsilon}\right)+f_{2}\left(t_{\varepsilon}\right)<f_{1}\left(t_{\varepsilon}\right) \leq f_{1}(1)=c_{1} .
$$

Thus Lemma4.1 is proved.

We are now in a position to prove Theorem 1.1

Proof of Theorem 1.1. As before, we let for small positive $\varepsilon$,

$$
\Phi_{\varepsilon}(u)=\frac{1}{2} \int_{\Omega}|\nabla u|^{2} d x-\frac{\lambda}{p_{1}(\varepsilon)+1} \int_{\Omega} \frac{\left(u^{+}\right)^{p_{1}(\varepsilon)+1}}{|x|^{s_{1}}} d x-\frac{1}{p_{2}(\varepsilon)+1} \int_{\Omega} \frac{\left(u^{+}\right)^{p_{2}(\varepsilon)+1}}{|x|^{s_{2}}} d x,
$$

where $p_{1}(\varepsilon)=2^{*}\left(s_{1}\right)-1-\varepsilon$ and $p_{2}(\varepsilon)=2^{*}\left(s_{2}\right)-1-\left(\frac{2-s_{2}}{2-s_{1}}\right) \varepsilon$. By Lemma 4.1, there exists $u_{0} \in H_{0}^{1}(\Omega)$ such that $\Phi_{\varepsilon}\left(u_{0}\right) \leq 0$ and

$$
c_{\varepsilon}^{*}=\inf _{P \in \mathcal{P}} \max _{w \in P} \Phi_{\varepsilon}(w),
$$

where $\mathcal{P}$ is the class of all continuous paths in $H_{0}^{1}(\Omega)$ connecting 0 with $u_{0}$. Let $c_{1}$ be the positive constant defined by (4.2). Then Lemma 4.1 yields

$$
c_{\varepsilon}^{*}<c_{1},
$$


provided that $\varepsilon \in\left[0, \varepsilon_{0}\right]$ for some small $\varepsilon_{0}>0$. For $\varepsilon>0$, there exists a solution $u_{\varepsilon} \in H_{0}^{1}(\Omega)$ of

$$
\begin{aligned}
& \Delta u_{\varepsilon}+\lambda \frac{u^{p_{1}(\varepsilon)}}{|x|^{s_{1}}}+\frac{u^{p_{2}(\varepsilon)}}{|x|^{s_{2}}}=0 \quad \text { in } \Omega, \\
& u_{\varepsilon}=0, \quad \text { on } \partial \Omega,
\end{aligned}
$$

and $\Phi_{\varepsilon}\left(u_{\varepsilon}\right)=c_{\varepsilon}$. Similar to (3.2), we have

$$
\int_{\Omega}\left|\nabla u_{\varepsilon}\right|^{2} d x \leq C_{1}
$$

for some constant $C_{1}$ independent of $\varepsilon$. By passing to a subsequence if necessary, we may assume

$$
u_{\varepsilon} \rightarrow u \quad \text { in } \quad H_{0}^{1}(\Omega) .
$$

If $u \not \equiv 0$, then clearly $u$ is a solution of (1.1) and Theorem 1.1 is proved. So it remains to prove $u \neq \equiv 0$ in $\Omega$.

Suppose $u \equiv 0$. As in Section 3, there exists $x_{\varepsilon} \in \Omega$ such that

$$
u_{\varepsilon}\left(x_{\varepsilon}\right)=\max u_{\varepsilon}(x)=m_{\varepsilon} \rightarrow+\infty,
$$

and after a linear transformation on $y$, we have

$$
v_{\varepsilon}(y)=\frac{u_{\varepsilon}\left(x_{\varepsilon}+k_{\varepsilon} y\right)}{m_{\varepsilon}} \rightarrow v(y) \quad \text { in } \quad C_{l o c}^{2}\left(\mathbb{R}_{+}^{N}\right),
$$

where $k_{\varepsilon}=m_{\varepsilon}^{-\frac{2}{N-2}+\frac{\varepsilon}{2-s_{1}}}$ and $v$ satisfies

$$
\begin{aligned}
& \Delta v+\lambda \frac{v^{p_{1}}}{|y|^{s_{1}}}+\frac{v^{p_{2}}}{|y|^{s_{2}}}=0 \quad \text { in } \quad \mathbb{R}_{+}^{N}, \\
& v=0 \quad \text { on } \quad \mathbb{R}_{+}^{N} .
\end{aligned}
$$

Also by a direct computation, we have

$$
\liminf _{\varepsilon \rightarrow 0} \int_{\Omega}\left|\nabla u_{\varepsilon}\right|^{2} d x \geq \int_{\mathbb{R}_{+}^{N}}|\nabla v|^{2} d y
$$

and

$$
\lim _{\varepsilon \rightarrow 0} \int_{\Omega} \frac{u^{p_{2}(\varepsilon)+1}}{|x|^{s_{2}}} d x \geq \int_{\mathbb{R}_{+}^{N}} \frac{v^{p_{2}+1}}{|y|^{s_{2}}} d y
$$

By (3.2), we have

$$
c_{\varepsilon}^{*}=\left(\frac{1}{2}-\frac{1}{p_{1}(\varepsilon)+1}\right) \int_{\Omega}\left|\nabla u_{\varepsilon}\right|^{2} d x+\left(\frac{1}{p_{1}(\varepsilon)+1}-\frac{1}{p_{2}(\varepsilon)+1}\right) \int_{\Omega} \frac{u_{\varepsilon}^{p_{2}(\varepsilon)+1}}{|x|^{s_{2}}} d x .
$$

Thus, (4.5) and (4.6) yields

$$
c^{*}=\lim _{\varepsilon \rightarrow 0} c_{\varepsilon}^{*}=\left(\frac{1}{2}-\frac{1}{2^{*}\left(s_{1}\right)}\right) \int_{\mathbb{R}_{+}^{N}}|\nabla v|^{2} d y+\left(\frac{1}{2^{*}\left(s_{1}\right)}-\frac{1}{2^{*}\left(s_{2}\right)}\right) \int_{\mathbb{R}_{+}^{N}} \frac{v^{2^{*}\left(s_{2}\right)}}{\left|y^{s_{2}}\right|} d y=c_{1},
$$

which contradicts to (4.4). Hence, $u \neq \equiv 0$, and then Theorem 1.1 is proved. 


\section{The Case $s_{2}=0$}

As discussed in Introduction, equation (1.1) with $\lambda<0$ has no least-energy solutions. In this sectin, we will consider a perturbed equation from equation (1.1):

$$
\left\{\begin{array}{l}
\Delta u-\frac{u^{2^{*}(s)-1}}{|x|^{s}}+u^{p}+u^{\frac{N+2}{N-2}}=0 \text { in } \Omega, \\
u(x)>0 \text { in } \Omega \text { and } u(x)=0 \text { on } \partial \Omega .
\end{array}\right.
$$

Theorem 5.1. Suppose $2^{*}(s)-1<p<\frac{N+2}{N-2}$ and $N \geq 4$. Then equation (5.1) has a positive solution.

Proof. Let

$\Phi(u):=\frac{1}{2} \int_{\Omega}|\nabla u|^{2} d x+\frac{1}{2^{*}(s)} \int_{\Omega} \frac{\left(u^{+}\right)^{2^{*}(s)}}{|x|^{s}} d x-\frac{1}{p+1} \int_{\Omega}\left(u^{+}\right)^{p+1} d x-\frac{N-2}{2 N} \int_{\Omega}\left(u^{+}\right)^{\frac{2 N}{N-2}} d x$.

Choose $0 \neq x_{0} \in \Omega$ and

$$
v_{\mu}(x)=\phi(x)\left(\frac{\mu}{1+\mu^{2}\left|x-x_{0}\right|^{2}}\right)^{\frac{N-2}{2}}
$$

for large $\mu>0$, where $\phi(x)$ is a cut-off function near $x_{0}$. Then it is not difficult to show that

$$
\sup _{t \geq 0} \Phi\left(t v_{\mu}\right)=\Phi\left(t_{0} v_{\mu}\right)<\frac{1}{N} S_{N}^{\frac{N}{2}}
$$

provided that $2^{*}(s)-1<p<\frac{N+2}{N-2}$ and $\mu$ is sufficiently large. Let $u_{0}=t_{0} v_{\mu}$, and

$$
c_{*}=\inf _{P \in \mathcal{P}} \max _{w \in P} \Phi(w),
$$

where $\mathcal{P}$ is the class of continuous paths in $H_{0}^{1}(\Omega)$ connecting 0 and $u_{0}$. Then it is easy to see

$$
0<c_{*}<\frac{1}{N} S_{N}^{\frac{N}{2}}
$$

We claim: $c_{*}$ is a critical value for $\Phi$. By the deformation lemma (see lemma in []), there exists $u_{j} \in H_{0}^{1}(\Omega)$ such that

$$
\left\{\begin{array}{l}
\Phi\left(u_{j}\right)=c_{*}(1+o(1)), \\
\int_{\Omega}\left|\nabla u_{j}\right|^{2} d x+\int_{\Omega} \frac{\left(u_{j}^{+}\right)^{2^{*}(s)}}{|x|^{s}} d x-\int_{\Omega}\left(u_{j}^{+}\right)^{p+1} d x-\int_{\Omega}\left(u_{j}^{+}\right)^{\frac{2 N}{N-2}} d x=o(1)\left\|u_{j}\right\|_{H_{0}^{1}},
\end{array}\right.
$$

where $o(1) \rightarrow 0$ as $j \rightarrow+\infty$. By (5.2) and (5.3), we have

$$
\int_{\Omega}\left|\nabla u_{j}\right|^{2} d x \leq C
$$

for some constant $C$ independent of $j$. By passing to a subsequence if necessary, we assume

$$
\left\{\begin{array}{l}
u_{j} \rightarrow u \quad \text { in } H_{0}^{1}(\Omega) \\
u_{j} \rightarrow u \text { in } L^{p+1}(\Omega) .
\end{array}\right.
$$

If $u \neq 0$, then it is easy to see $u$ is a solution of (5.1) and $\Phi(u)=c_{*}$. Hence, it remains to show that $u \neq \equiv 0$. We prove it by contradiction. 
Now suppose $u \equiv 0$, and set

$A=\varliminf_{j \rightarrow+\infty} \int_{\Omega}\left|\nabla u_{j}\right|^{2} d x, \quad B=\underline{\lim }_{j \rightarrow+\infty} \int_{\Omega} \frac{\left(u_{j}^{+}\right)^{2^{*}(s)}}{|x|^{s}} d x$ and $C=\varliminf_{j \rightarrow+\infty} \int_{\Omega}\left(u_{j}^{+}\right)^{\frac{2 N}{N-2}} d x$.

Then (5.3) implies

$$
c_{*}=\frac{A}{2}+\frac{B}{2^{*}(s)}-\frac{N-2}{2 N} C
$$

and

$$
C=A+B
$$

By the Sobolev inequality, (5.5) implies

$$
C=A+B \geq A \geq S_{N} C^{1-\frac{2}{N}} .
$$

Thus, we have

$$
C \geq S_{N}^{\frac{N}{2}} \text { and } A \geq S_{N} C^{1-\frac{2}{N}} \geq S_{N}^{\frac{N}{2}}
$$

Then (5.4) yields

$$
c_{*}=\left(\frac{1}{2}-\frac{N-2}{2 N}\right) A+\left(\frac{1}{2^{*}(s)}-\frac{N-2}{2 N}\right) B \geq \frac{1}{N} S_{N}^{\frac{N}{2}},
$$

a contradiction to (5.2). Hence, Theorem5.1 is proved.

\section{References}

[1] T. Bartsch, S. Peng and Z. Zhang, Existence and non-existence of solutions to elliptic equations related to the Caffarelli-Kohn-Nirenberg inequalities, Calc. Var. Partial Differential Equations 30 (2007), no. 1, 113-136.

[2] H. Berestycki and L. Nirenberg, Monotonicity, symmetry and antisymmetry of solutions of semilinear elliptic equations, J. Geom. Phys. 5 (1988), no. 2, 237-275.

[3] H. Brézis and L. Nirenberg, Positive solutions of nonlinear elliptic equations involving critical Sobolev exponents, Comm. Pure Appl. Math. 36 (1983), no.4, 437-477.

[4] L. Caffarelli, R. Kohn and L. Nirenberg, First order interpolation inequalities with weights, Compositio Math. 53 (1984), no. 3, 259-275.

[5] D. Cao and P. Han, Solutions to critical equation with multi-singular inverse square potentials, J. Differential Equations 224 (2006), no. 2, 332-372.

[6] F. Catrina and Z. Q. Wang, On the Caffarelli-Kohn-Nirenberg inequalities: sharp constants, existence (and nonexistence), and symmetry of extremal functions, Comm. Pure Appl. Math. 54 (2001), no.2, 229-258.

[7] C. C. Chen and C. S. Lin, A spherical Harnack inequality for singular solutions of nonlinear elliptic equations, Ann. Scuola Norm. Sup. Pisa Cl. Sci. (4) 30 (2001), (2002), no.3-4, 713-738.

[8] C. C. Chen and C. S. Lin, Estimates of the conformal scalar curvature equation via the method of moving planes, Comm. Pure Appl. Math. 50 (1997), no.10, 971-1017.

[9] C. C. Chen and C. S. Lin, Estimate of the conformal scalar curvature equation via the method of moving planes. II, J. Differential Geom. 49 (1998), no. 1, 115-178. 
[10] C. C. Chen and C. S. Lin, Local behavior of singular positive solutions of semilinear elliptic equations with Sobolev exponent, Duke Math. J. 78 (1995), no. 2, 315-334.

[11] J. L. Chern and C.S. Lin, Minimizers of Caffarelli-Kohn-Nirenberg inequalities on domains with the singularity on the boundary, Arch. Rational Mech. Anal. 197 (2010), 401-432.

[12] K. S. Chou and C. W. Chu, On the best constant for a weighted Sobolev-Hardy inequality, J. London Math. Soc. (2) 48 (1993), no. 1, 137-151.

[13] J. Dolbeault, M. J. Esteban and G. Tarantello, The role of Onofri type inequalities in the symmetry properties of extremals for Caffarelli-Kohn-Nirenberg inequalities, in two space dimensions, Ann. Sc. Norm. Super. Pisa Cl. Sci. (5) 7 (2008), no. 2, 313-341.

[14] N. Ghoussoub and X. S. Kang, Hardy-Sobolev critical elliptic equations with boundary singularities, Ann. Inst. H. Poincaré Anal. Non Linéaire 21 (2004), no.6, 767-793.

[15] N. Ghoussoub and F. Robert, The effect of curvature on the best constant in the HardySobolev inequalities, Geom. Funct. Anal. 16 (2006), no.6, 1201-1245.

[16] B. Gidas, W. M. Ni and L. Nirenberg, Symmetry of positive solutions of nonlinear elliptic equations in $\mathbb{R}^{n}$, Mathematical analysis and applications, Part A, pp. 369-402, Adv. in Math. Suppl. Stud., 7a, Academic Press, New York-London, 1981.

[17] C. H. Hsia, C. S. Lin and H. Wadade, Revisiting an idea of Brézis and Nirenberg, to appear in J. Funct. Anal..

[18] Q. Jin, Y. Y. Li and H. Xu, Symmetry and Asymmetry: The method of moving spheres, Advances in Differential Equations 13 (2008), 601-640.

[19] Y. Y. Li, Prescribing scalar curvature on $S^{n}$ and related problems. I, J. Differential Equations 120 (1995), no. 2, 319-410.

[20] Y. Y. Li and L. Zhang, Liouville type theorems and Harnack type inequalities for semilinear elliptic equations, Journal d'Analyse Mathematique 90 (2003), 27-87.

[21] Y. Y. Li and M. Zhu, Uniqueness theorems through the method of moving spheres, Duke Math. J. 80 (1995), 383-417.

[22] E. H. Lieb, Sharp constants in the Hardy-Littlewood-Sobolev and related inequalities, Ann. of Math. 118 (1983), no.2, 349-374.

[23] C. S. Lin, Interpolation inequalities with weights, Comm. Partial Differential Equations 11 (1986), no. 14, 1515-1538.

[24] C. S. Lin and Z. Q. Wang, Symmetry of extremal functions for the Caffarelli-KohnNirenberg inequalities, Proc. Amer. Math. Soc. 132 (2004), no.6, 1685-1691.

[25] R. Musina, Ground state solutions of a critical problem involving cylinderical weights, Nonlinear Anal. 68 (2008), no. 12, 3972-3986.

[26] J. Serrin, A symmetry problem in potential theory, Arch. Ration. Mech. 43 (1971), 304-318.

\author{
YanYan Li \\ Department of Mathematics \\ Rutgers University \\ Piscataway, NJ 08854, USA \\ yyli@math.rutgers.edu
}

\section{Chang-Shou Lin}

Taida Institute for Mathematical Sciences

Department of Mathematics

National Taiwan University

Taipei 106, Taiwan

cslin@math.ntu.edu.tw 